\title{
A note on magnetic vector potential
}

\author{
Raghavendra G. Kulkarni ${ }^{*}$
}

\begin{abstract}
This paper presents a non-traditional way of determining an unknown constant encountered in the expression for magnetic vector potential due to an elemental dipole antenna.

K e y w o r d s: infinitesimal current element, Hertzian dipole antenna, magnetic vector potential, Lorentz gauge condition, Helmholtz wave equation, divergence theorem, volume integral, surface integral
\end{abstract}

\section{Introduction}

We briefly review the derivation of the expression for magnetic vector potential due to a Hertzian dipole antenna, with an emphasis on the evaluation of a constant encountered in the expression. Then we describe a new method of evaluation of this constant, which is normally not dealt in the textbooks.

The concepts of time varying magnetic vector potential, $\boldsymbol{A}$, and the electric scalar potential, $V$, are invariably introduced in the very first few lectures while teaching a basic course on antennas $[1,2,3,4,5,6]$. The time varying magnetic flux density, $\boldsymbol{B}$, is determined from the curl operation on magnetic vector potential, $i e, \boldsymbol{B}=\nabla \times \boldsymbol{A}$. The time varying electric field intensity, $\boldsymbol{E}$, is obtained from the relation, $\boldsymbol{E}=-\nabla V-\dot{\boldsymbol{A}}$. Elimination of electric and magnetic field terms from the Maxwell equations using the above mentioned relations between the potentials and the fields results in two coupled partial differential equations in $\boldsymbol{A}$ and $V$, which are difficult to solve. The introduction of Lorentz gauge condition in the coupled equations leads to the two uncoupled in-homogeneous Helmholtz wave equations in $\boldsymbol{A}$ and $V$. For sinusoidal time variations, these Helmholtz equations become,

$$
\begin{aligned}
\left(\nabla^{2}+\omega^{2} \mu \varepsilon\right) \boldsymbol{A} & =-\mu \boldsymbol{J}, \\
\left(\nabla^{2}+\omega^{2} \mu \varepsilon\right) V & =-\rho / \varepsilon,
\end{aligned}
$$

where $\boldsymbol{J}$ is the current density; $\omega, \mu, \varepsilon$ and $\rho$ have the usual meaning as described in [1]. Many textbooks introduce $z$-directed infinitesimal current element $(I \mathrm{~d} l)$ placed at origin as an elemental antenna, or, Hertzian dipole antenna, and proceed to determine the potential, $\boldsymbol{A}$, due to this source. As a result, the in-homogeneous Helmholtz wave equation (in potential $\boldsymbol{A}$ ) reduces to a scalar equation as shown below,

$$
\left(\nabla^{2}+k^{2}\right) A_{z}=-\mu J_{z}
$$

where $A_{z}$ is the scalar part of $z$-directed magnetic vector potential, $k^{2}=\omega^{2} \mu \varepsilon$ and $J_{z}$ is the scalar part of $z$ directed current density. At any point $\mathrm{P}$ in the source-free field region, see Fig. 1, equation (2) reduces to,

$$
\left(\nabla^{2}+k^{2}\right) A_{z}=0
$$

which is homogeneous Helmholtz equation. Expressing $\nabla^{2} A_{z}$ in spherical coordinate system, and noting that there is no variation of $A_{z}$ in $\theta$ and $\phi$ directions, the solution of (3) is obtained as,

$$
A_{z}=\frac{C}{r} e^{-j k r}
$$

where $r$ is the radial distance of $\mathrm{P}$ from the origin; and $\mathrm{C}$ is an unknown constant. The textbooks deal with either of the following two methods for evaluation of $\mathrm{C}$.

The first method makes use of the similarity that exists between the inhomogeneous Helmholtz wave equation (2) when $k \rightarrow 0$ and the Poisson's equation,

$$
\nabla^{2} V=-\rho / \varepsilon
$$

for the electrostatic potential, $V$. Notice that $k \rightarrow 0$ corresponds to $\omega \rightarrow 0$, which is electrostatic case $[3,4$, $5,6]$. A comparison with the solution of the Poisson's equation, for the case of a point charge placed at origin, leads to evaluation of $C$ as

$$
C=\frac{\mu I \mathrm{~d} l}{4 \pi}
$$

The second method employs divergence theorem for the evaluation of $C$ as described below $[1,2]$.

Consider an infinitesimal spherical volume centered at the origin having radius $r_{0}$, enclosing the current element $I \mathrm{~d} l$. Now, consider (2), which includes the source term. Taking the volume integral of (2) within the in

* Department of Electronics and Communication Engineering, PES University, 100 Feet Ring Road, BSK III Stage, Bengaluru-560085, India,dr_rgkulkarni@yahoo.com, raghavendrakulkarni@pes.edu 
nitesimal sphere results into,

$$
\int_{v} \nabla^{2} A_{z} \mathrm{~d} v+\int_{v} k^{2} A_{z} \mathrm{~d} v=-\int_{v} \mu J_{z} \mathrm{~d} v
$$

As the infinitesimal spherical volume starts shrinking (and in the limit becomes zero), the radius $r_{0}$; also tends to zero. So, applying this limit, in (6), the right-hand-side is determined as $-\mu I \mathrm{~d} l$; and since $d v=r^{2} \sin \theta \mathrm{d} \theta \mathrm{d} \phi \mathrm{d} r$ the second term vanishes. Thus we have,

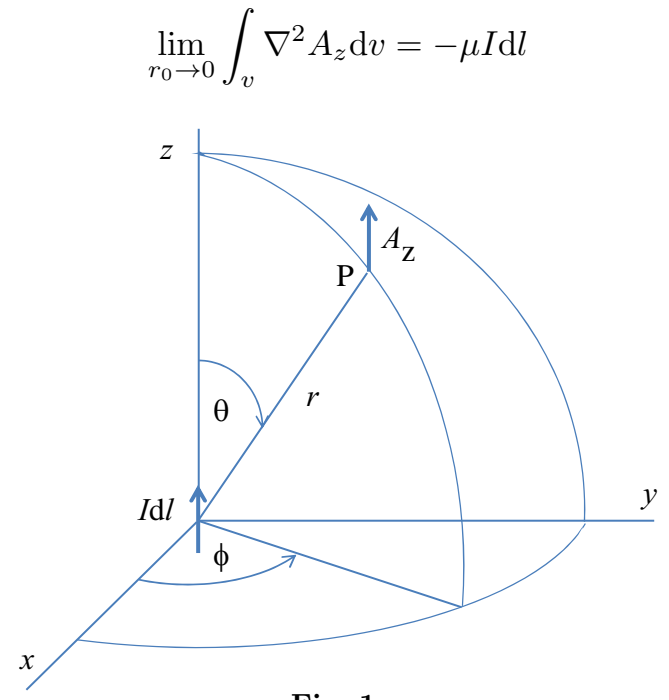

Fig. 1.

Rewriting $\nabla^{2} A_{z}$ as $\nabla \cdot \nabla A_{z}$, and applying divergence theorem to convert the volume integral the above expression to a closed surface integral leads to,

$$
\int_{v} \nabla \cdot \nabla A_{z} \mathrm{~d} v=\oint_{s} \nabla A_{z} \cdot \mathrm{d} \mathbf{s}
$$

and further it becomes,

$$
\lim _{r_{0} \rightarrow 0} \oint_{s} \nabla A_{z} \cdot \mathrm{d} \mathbf{s}=-\mu I \mathrm{~d} l
$$

where $\mathrm{d} \boldsymbol{s}=\mathbf{a}_{r} r_{0}^{2} \sin \theta \mathrm{d} \theta \mathrm{d} \phi$; and because of spherical symmetry $\nabla A_{z}$ reduces to: $\nabla A_{z}=\mathbf{a}_{r} \partial A_{z} / \partial r$ : Therefore

$$
\nabla A_{z} \cdot \mathrm{d} \boldsymbol{s}=r_{0}^{2} \sin \theta\left(\partial A_{z} / \partial r\right) \mathrm{d} \theta \mathrm{d} \phi
$$

and since the integration is over the closed spherical surface of radius $r=r_{0}$,

$$
\left.\frac{\partial A_{z}}{\partial r}\right|_{r=r_{0}}=-\frac{C}{r_{0}^{2}}\left(1+j k r_{0}\right) e^{-j k r_{0}}
$$

and so (7) can be written as,

$$
\lim _{r_{0} \rightarrow 0} \oint_{s} C \sin \theta\left(1+j k r_{0}\right) e^{-j k r_{0}} \mathrm{~d} \theta \mathrm{d} \phi=\mu I \mathrm{~d} l
$$

After the integration and the application of the limit, (8) becomes, $4 \pi C=\mu I d l$; yielding again (5).

A third method to evaluate $C$ is presented in the next section, which is normally not dealt in the textbooks.

\section{New method}

A new method to determine $\mathrm{C}$, which does not make use of the divergence theorem for the volume integral in (6) is described below.

Since the potential, $A_{z}$, has no variation in $\theta$ and $\phi$ directions, $\nabla^{2} A_{z}$ is expressed as [1]:

$$
\nabla^{2} A_{z}=\frac{1}{r^{2}} \frac{\partial}{\partial r}\left(r^{2} \frac{\partial A_{z}}{\partial r}\right) .
$$

Use of the above expression in (6) and applying the limit leads to

$$
\lim _{r_{0} \rightarrow 0} \int_{v} \frac{\partial}{\partial r}\left(r^{2} \frac{\partial A_{z}}{\partial r}\right) \sin \theta \mathrm{d} \theta \mathrm{d} \phi \mathrm{d} r=-\mu I \mathrm{~d} l
$$

Since $\left(\partial A_{z} / \partial r\right)=-C(1+j k r) \exp (-j k r) / r^{2} ;$ using it in (9), and after the double integration with $\theta \in(0, \pi)$ and $\phi \in(0,2 \pi)$, one obtains,

$$
\lim _{r_{0} \rightarrow 0} 4 \pi C \int \frac{\partial}{\partial r}(1+j k r) e^{-j k r_{0}} \mathrm{~d} r=\mu I \mathrm{~d} l
$$

Note that integration of a differentiation of a function is the function itself, and since $r \leq r_{0}$, as $r_{0} \rightarrow 0$ we have $r \rightarrow 0$, so

$$
\lim _{r \rightarrow 0} 4 \pi C(1+j k r) e^{-j k r}=\mu I \mathrm{~d} l
$$

and after applying the limit, one gets the same result (5) as before.

\section{Conclusion}

A brief review of derivation of expression for magnetic vector potential, $\boldsymbol{A}$, is given; the two traditional methods to evaluate a constant $(C)$ encountered in the expression for $\boldsymbol{A}$ are discussed along with a new method presented in this paper. The new method will provide the readers a broader insight into the subject beyond what they learn from the textbooks.

\section{Acknowledgements}

The author thanks the management of PES University, Bengaluru, for supporting this work.

\section{REFERENCES}

[1] Robert E. Collin, Antennas and Radio Wave Propagation, Mc-Graw Hill International Student Edition, 1985.

[2] Robert E. Collin, Foundations for Microwave Engineering, Wiley (India), 2nd Edition, 2005.

[3] Edward C. Jordan and Keith G. Balmain, Electromagnetic Waves and Radiating Systems, Prentice Hall Inc., New Delhi, 2nd Edition, 2009.

[4] A. R. Harish and M. Sachidananda, Electromagnetic Waves and Radiating Systems, Oxford University Press, New Delhi, 2007.

[5] Constantine A. Balanis, Antenna Theory, Wiley-India, 3rd Edition, 2005.

[6] Roger F. Harrington, Time-harmonic electromagnetic fields, IEEE Press, 2001, A classic reissue of original (1961) Edition.

Received 31 October 2017 